\title{
TRAJEKTE
}

Eine Reihe des Zentrums für

Literatur- und Kulturforschung Berlin

Herausgegeben von

Sigrid Weigel und Karlheinz Barck $(\dagger)$ 


\section{Klang und Musik bei Walter Benjamin}

Tobias Robert Klein

in Verbindung mit Asmus Trautsch 
Die dieser Publikation zugrundeliegende Tagung wurde vom Bundesministerium für Bildung und Forschung unter dem Förderkennzeichen 01UG0712 gefördert. Die Verantwortung für den Inhalt liegt bei den Autoren.

$$
\text { Umschlag: }
$$

Nach dem Plakatentwurf von Carolyn Steinbeck · Gestaltung, Berlin

Bibliografische Information der Deutschen Nationalbibliothek

Die Deutsche Nationalbibliothek verzeichnet diese Publikation in der Deutschen Nationalbibliografie; detaillierte bibliografische Daten sind im Internet über http://dnb.d-nb.de abrufbar.

Alle Rechte, auch die des auszugsweisen Nachdrucks, der fotomechanischen Wiedergabe und der Übersetzung, vorbehalten. Dies betrifft auch die Vervielfältigung und Übertragung einzelner Textabschnitte, Zeichnungen oder Bilder durch alle Verfahren wie Speicherung und Übertragung auf Papier, Transparente, Filme, Bänder, Platten und andere Medien, soweit es nicht $\$ \$ 53$ und 54 UrhG ausdrücklich gestatten.

(C) 2013 Wilhelm Fink Verlag, München

Wilhelm Fink GmbH \& Co. Verlags-KG, Jühenplatz 1, D-33098 Paderborn

Internet: www.fink.de

Redaktion und Lektorat: Bettina Moll, Berlin Einbandgestaltung: Evelyn Ziegler, München

Printed in Germany.

Herstellung: Ferdinand Schöningh GmbH \& Co KG, Paderborn

ISBN 978-3-7705-5343-3 


\section{ELI FriedLander}

\section{Farben und Laute in der Berliner Kindheit um neunzehnhundert}

In Benjamins Berliner Kindheit um neunzehnhundert zitiert das Sich-Erinnern an Erfahrenes häufig sensorische Qualitäten herbei. In der vorliegenden Abhandlung möchte ich die Schilderung der Farben- und Klangräume in der Weise beleuchten, wie sie die Erfahrungswelt der Kindheit mitprägten. Ferner möchte ich untersuchen, wie diese in die Erinnerung überführt und dort zusammengefügt wurden. Des Weiteren habe ich die Absicht, der Frage nachzugehen, in welcher Weise diese Erfahrungsregister an der Realisierung der Vergangenheit in der Erinnerung mitwirkten. Demnach werde ich versuchen, erstens spezifische Textmomente zu interpretieren, in denen die Überführung von Farben und Klängen in die Erinnerung wesentlich ist, und zweitens zu ergründen, inwiefern für Benjamin solche Momente Allegorien der Erinnerung darstellen. ${ }^{1}$

\section{Zwei Traumbilder}

Beginnen möchte ich mit einer allgemeinen, das Thema Erinnerung in diesem Text betreffenden Bemerkung, die ich in Bezug auf zwei Momente der Berliner Kindheit veranschaulichen möchte. In ihrer Überschrift führt Benjamins Berliner Kindheit um neunzehnhundert Zeit, Raum und eine spezifische Erfahrungswelt zusammen: die Kindheit in dieser Stadt zu dieser Zeit. Eine derartige Titelstruktur könnte auf die Einbindung der Lebenswelt in den Er-

1 Meine Erörterung des Stellenwerts, den Benjamin den Farben zumisst, ist in Teilen einer längeren Untersuchung entnommen, die inzwischen unter dem Titel »A Mood of Childhood in Benjamin« in: Hagi Kenan/Ilit Ferber (Hg.): Philosophy's Mood. On the Affective Grounds of Thinking, Berlin (Springer) 2011, S.39-50 erschienen ist. fahrungsraum des Einzelnen deuten. Darüber hinaus verweist dies möglicherweise auf eine zu erschließende Ebene oder Dimension in der Erinnerung des Einzelnen, in der sich diese lebensweltlichen Umgebungen selber brechen. Weil Umgebungen dem Kind aber keine Gegenstände von Einbeziehung und Aufmerksamkeit sein können, mag man sich fragen, wie sie denn überhaupt in der Erinnerung aufscheinen können. Und in der Tat, was hieße es für uns, die Umgebungen der Kindheit in Erinnerung zu rufen? Der Gedanke, die Wahrnehmung benötigen einen Gegenstand; Umgebungen jedoch wären zu unscharf, um ihrer habhaft zu werden. Je tiefer die Ebene der wiederbelebten Erfahrung ist, je unabhängiger von bestimmten Gesichtern, Namen, Ereignissen und Geschichten, desto weniger scheint es darüber hinaus in unserer Hand zu liegen, diese zu vergegenwärtigen.

Wichtig ist, dass Benjamin nicht meint, er erinnere sich an verschiedene in der Kindheit wahrgenommene Bilder, quasi im Sinne der Vergegenwärtigung früher gesehener Dinge. Benjamin spricht davon, auf welche Weise sich »die Erfahrung der Großstadt in einem Kinde der Bürgerklasse [...] niederschlägt" (GS VII, 385). Mit anderen Worten ist ein Bild im hier verwendeten Sinne nicht das Korrelat einer früheren Wahrnehmung, sondern etwas, das allein in der Erinnerung erscheint, etwas, das Umgebungen bedeutet und erfasst, die möglicherweise keine intentionalen Gegenstände des Bewusstsein sind.

Das Wort »Niederschlag«, das Benjamin hier verwendet, um das In-Erscheinung-Treten der Bilder zu kennzeichnen, deutet darauf hin, dass durch die Anhäufung von Erfahrung und die Sättigung der Erinnerung die Erfahrungsmasse schließlich kondensieren, ihren Aggregatszustand ändern oder in einem Bild ihren Niederschlag finden mag. "Niederschlag« zitiert das Wetter herbei, insbesondere jene Regentage, die u. a. wegen ihrer allumfassenden Eigenschaft von Belang sind, die der Regen der Stadt verleiht. Regen taucht sie in ein uniformes, facettenloses Grau, bringt sie als eine Umgebung hervor, verschafft ihr eine Stimmung, die sogar das eigene Zeitempfinden ändert (an Regentagen kann man, wie Benjamin schreibt, vom »Morgen bis zum Abend [...] dasselbe tun" [GS V, 159]). Das Bild jedoch, in welchem diese ganze Umgebung wirklich ihren Niederschlag findet, hat keine ersichtliche Ähnlichkeit mit ihr. Der Fischotter ist »das heilige Tier des Regenwassers" und als solches scheint er die regnerische Umgebung gleichsam in sich aufzusaugen. "Wenn ich in sein Wasser blickte«, so Benjamin, "war mir immer, als stürze Regen in alle Gullys der 
Stadt, nur um in dieses Becken zu münden und sein Tier zu speisen ... Ob es aber in diesen Abwässern und Wässern sich gebildet habe oder von seinen Strömen und seinen Rinnseln sich nur speise, hätte ich nicht entscheiden können.« Endlos wartete das Kind, um den "gleißende[n] Insasse[n] der Zisterne" - und sei es auch nur für einen Moment - zu erblicken. Huschte das Tier dann einmal zur Oberfläche, so nur, um einen Augenblick später wieder »in der nassen Nacht« zu verschwinden - "als wenn es in seiner Tiefe unentbehrlich sei« (GS VII, 407 f.). Warten und Langeweile sind in diesem Vorgefühl, diesem Wunschbild konzentriert, das zugleich als Allegorie der Notwendigkeit des Wartens dient, der Bürde oder Macht der Vergangenheit, die sich in einem flüchtigen, vergänglichen Erinnerungsbild Ausdruck verleiht.

Nehmen wir ein anderes Erinnerungsbild: das der Schmetterlingsjagd. „Es begann«, so schreibt Benjamin, »die alte Jägersatzung zwischen uns zu herrschen: je mehr ich selbst in allen Fibern mich dem Tier anschmiegte, je falterhafter ich im Innern wurde, desto mehr nahm dieser Schmetterling in Tun und Lassen die Farbe menschlicher Erschließung an und endlich war es, als ob sein Fang der Preis sei, um den einzig ich meines Menschendaseins wieder habhaft werden könne« (392). Nicht allein wird aus der metamorphorischen Kreatur par excellence das Objekt einer mimetischen Verwandlung des Kindes, sondern diese Identifikation ist es, die eine tiefere, gestaltlosere Aneignung des Umfelds stützt. Über den Flug der Schmetterlinge gebiete »die Verschwörung von Wind und Düften, Laub und Sonne" (ebd.). Das Wort "Verschwörung" lässt vermuten, dass die Elemente durch irgendeinen geheimen Plan zusammenfinden, dessen Bild der Schmetterling ist. In dem ephemeren Geschöpf verschränken sich diese verschiedenen unfassbaren Dimensionen, sein Flug ermöglicht ihnen, Zugang zum Kind zu finden. Sicher ließen sich die Bewegungen des Schmetterlings unter Rückgriff auf die verschiedenen Faktoren beschreiben, welchen sie in Raum und Zeit ursächlich ausgesetzt sind; der Vorstellungsraum jedoch erschließt sich durch ein Bild - einem Zusammenspiel dessen, was womöglich weder physisch noch psychisch ist. Ein einzelner schillernder Gegenstand wird in seiner Vorstellung von einem Netz überzogen, dessen verschiedene Maschen eine Vielzahl unterschiedlicher Dinge versammeln. Deren Verwandtschaft ist keine Sache von Ähnlichkeit, Vorstellung und metaphorischer Übertragung, sondern hat im Bild ihr Zentrum. Vermöge dieses impliziten Umfelds an Bedeutung erlangt das Bild seine beson- dere Signifikanz und Anziehungskraft. Es verleiht ihm etwas, das Benjamin in einem andern Zusammenhang 'Aura nennt, und lässt einen Blick erwidern, der uns die Aufmerksamkeit, die wir ihm schenken, mit Bedeutung belohnt - wie auch der Schmetterling, wenn das Kind ihn verfolgt, »die Farbe menschlicher Erschließung " (ebd.) anzunehmen scheint.

Es gibt hier also zwei Bilder, ein Bild des Winters und eines des Sommers (auf den jahreszeitlichen Aspekt von Erfahrung werde ich zu einem späteren Zeitpunkt in meiner Auseinandersetzung mit der Musik zurückkommen). Es ließe sich auch behaupten, das eine sei ein Bild von Entfärbung oder Düsterkeit, das andere ein solches der Farbenpracht. Dies veranlasst mich, nach dem Ort und der Bedeutung von Farbe in der Welt der Kindheit zu fragen.

\section{Farbe und Phantasie}

"Die Gegenständlichkeit der Farbe«, so Benjamin in einem frühen Fragment mit dem Titel "Die Farbe vom Kinde aus betrachtet«, »hebt die intellektuellen Verbindungen der Seele auf und schafft die reine Stimmung« (GS VI, 111). Von subjektiven Empfindungen und Gefühlszuständen sind Stimmungen u.a. dadurch zu unterscheiden, dass sie affektive Manifestationen des eigenen Umfelds und der eigenen Situiertheit in ihm sind. Stimmungen künden von unserem Einklang mit diesen Zusammenhängen oder der Entzweiung von ihnen. Um eine Stimmung zu erzeugen, darf Farbe nicht als eine Eigenschaft einzelner Gegenstände erscheinen, sondern hat die Textur der Erfahrung als eines Ganzen hervorzukehren, das mit seinem Bewusstsein eins ist. Damit ist nicht gemeint, man müsse sich, um eine solche Stimmung zu erfahren, künstlich auf den subjektiven Raum der eigenen Sinneseindrücke konzentrieren (eine solche Haltung würde, wenn überhaupt, nur dazu dienen, die Stimmung zu verschleiern, in der man sich gerade befinden mag). Stimmungen sind keine Achtsamkeit erheischenden Gegenstände, auch verlangt ihre Bekundung keine Aufmerksamkeit. Das Kind kann sich im Einklag mit der Farbe finden, wenn es geistesabwesend, in ihr verloren oder in sie versunken ist, wenn es sich verschiedensten Beschäftigungen und Ablenkungen hingibt.

Die Art, wie Farbe als Stimmung erfahren, als Umgebung aufgenommen werden kann, entspricht dem, was Benjamin allgemeiner mimetisches Vermö- 
gen nennt. Das Kinderspiel ist ihm dafür eine der deutlichsten Manifestationen. Dieses »ist überall durchzogen von mimetischen Verhaltungsweisen; und ihr Bereich ist keineswegs auf das beschränkt, was ein Mensch dem anderen nachmacht. Das Kind spielt nicht nur Kaufmann oder Lehrer, sondern auch Windmühle und Eisenbahn.» (GS II, 209) In Zusammenhängen wie diesen sprechen wir von kindlichem Vorstellungsvermögen. Andererseits können Kinder nicht Farbe spielen. "Aller Form nämlich, allem Umriß, den der Mensch wahrnimmt, entspricht er selbst in dem Vermögen, ihn hervorzubringen. [...] Dieses Vermögen aber hat an der Welt der Farbe seine Grenze; der Menschenkörper kann die Farbe nicht erzeugen. Er entspricht ihr nicht schöpferisch, sondern empfangend: im farbig schimmernden Auge." (GS IV, 613) Somit bildet Farbe den Grenzfall des Mimetischen, der, wie das für Grenzfälle allgemein gilt, diesem Vermögen etwas Wesentliches zum Vorschein bringt. Charakteristischere Formen mimetischen Verhaltens gründen in der inneren Beziehung des Einzelnen zu seiner Welt. In der Erfahrung der Kindheit zeigt sich diese Art der Zugehörigkeit des Einzelnen zur Welt in besonders deutlicher Weise.

Um die Rezeptivität der Farbe von den aktiven Formen vorgestellter Identifikation zu unterscheiden, erinnert Benjamin in einem mit "Die Farben" betitelten Abschnitt der Berliner Kindheit an eine abwechslungsreiche Verwandlung, die dem Kind geschah, als es in einem verlassenen und morschen Gartenpavillon mit bunten Fenstern umherging: »Wenn ich in seinem Innern von Scheibe zu Scheibe strich, verwandelte ich mich; ich färbte mich wie die Landschaft, die bald lohend und bald verstaubt, bald schwelend und bald üppig im Fenster lag.» (GS VII, 424) Das Bild des Versenktseins in Farbe offenbart einen grundlegenden Unterschied zwischen der Rolle, welche Farbe in der Welt des Kindes spielt, und ihrem Auftreten in der Kunst. Man kann sich bei der Betrachtung eines Bildes in dieses vertiefen, das Kind jedoch vertieft sich in die Farbe, als ob sie seine Umgebung wäre. ${ }^{2}$ Dieser Versenkung Ver-

2 Die Unterscheidung zwischen der Versenkung in das Kunstwerk und der gegensätzlichen Haltung, das Kunstwerk im Betrachter zu versenken, ist von grundlegender Bedeutung für Benjamins Verständnis des Unterschieds zwischen traditionellem ästhetischen Urteil und dessen verwandelter Erscheinung im Film. Vgl. zu diesem Zusammenhang "Das Kunstwerk im Zeitalter seiner technischen Reproduzierbarkeit« (GS I, 571-508, insb. 465). gleichbares geschieht beim »Tuschen«: »[D]ie Dinge [taten] mir ihren Schoß auf]], sobald ich sie mit einer feuchten Wolke überkam« (ebd.). Die Farbe erweitert, enthüllt und gibt sich gleichsam hin, statt als Erweiterung der Malhandlung zu dienen. Diese überraschende Wendung der Handlung zur bloßen Gebärde ergreift den Blick der Phantasie und nimmt das Kind gefangen. ${ }^{3}$

Obwohl Farbe aufgenommen wird, darf sie doch nicht mit dem bloßen Material des sinnlich Gegebenen verwechselt werden. Die Freude an der Farbe ist keine Sache ihrer angenehmen Wirkung. Wird sie eine Stimmung genannt, so nicht deshalb, weil sie etwa die Annehmlichkeit einer Umgebung bewirkte. Farbe vermittelt uns Erfahrungen, ohne aber in ihrer Erscheinung verführerisch zu sein. Denn sie verspricht nichts jenseits ihrer selbst. Farbe vermag auf die gewöhnlichste und nüchternste Weise zu berauschen und verweist so auf ihre geistige Natur. An eine derart 'profane Erleuchtung erinnert Benjamin im zweiten Teil des 'Farben«-Abschnitts, der den Wettkampf zwischen höheren und niederen Sinnen thematisiert. Dieser entwickelt sich, während das Kind einen Goldfaden entknotet, der Schokoladenpäckchen zusammenhält. Jedes dieser Stücke war für sich in farbiges Stanniolpapier verpackt, grün und golden, blau und orange, rot und silber - ein kleines, prächtiges Bauwerk, dessen Zusammensetzung allein farblich geordnet war, nämlich dem Prinzip

3 "Diese selbstgenügsam prangende Farbenwelt« (GS III, 18) der Kinderbücher - deren leidenschaftlicher Sammler Benjamin war - bietet eine weitere Gelegenheit, den Unterschied zur Malerei zu entfalten. In der Malerei ist Farbe ein Element der Form und gehört zu den Möglichkeitsbedingungen des artistischen Mediums. Während Gemälde aber teilweise als aus ihren Farben zusammengesetzt wahrgenommen werden, wird Farbe selbst nicht als etwas erfahren, das irgendetwas überdeckt, weder einen Hintergrund noch eine andere Farbe. Stattdessen gehört sie in der gleichen Weise zum Kunstwerk wie das Erröten oder Erbleichen zu einem Seelenzustand. Hingegen wurde bei den Illustrationen alter Kinderbücher zuerst die graphische Kontur gedruckt. Die Farbgebung erfährt man, oft in ihren Fehlern und Mängeln, als aufgedruckt oder über den Linien schwebend. Man kann über die wundervolle Farbgebung dieser Illustrationen nicht genug staunen. Hingegen über ein Gemälde zu sagen, es habe wundervolle Farben, verriete nicht nur mangelnden Geschmack, sondern hieße, einen Kategorienfehler zu begehen. Wer in der Malerei die Farbe von der Gestalt oder Form sondert, riskiert, sie zum bloßen Effekt herabzusetzen. Illustrationen hingegen bergen nicht die Gefahr, dass die Farbe das Kommando übernimmt. Weil Illustrationen in Kinderbüchern mit der Schrift die horizontale Ebene teilen, würde es ihrer Bedeutung widersprechen, sie wie ein Gemälde - vertikal - zu betrachten, als etwas, das ausgestellt wird. 
folgend, dass keine Stücke der gleichen Farbe einander berühren durften. Der Wettkampf zwischen den Sinnen, zwischen Mund und Auge, ist entschieden, indem die Freude an der Schokolade dadurch gereinigt, man könnte sagen: vergeistigt wird, dass sie in der Erfahrung der Farben aufgeht. Während die Schokolade im Mund schmilzt, wird ihre Süße aufgegriffen, aufgehoben im verwirrenden Erinnerungsbild einer eher im Herzen als auf der Zunge zergehenden Süßigkeit.

Die geistige Natur der Farbe ist damit verbunden, dass sie nicht als Anzeichen eines von ihr Unterschiedenen erscheint: Klänge oder Gerüche führen zurück zu einer unabhängig von ihnen beschreibbaren Quelle; Geschmack und taktil erfassbare Qualitäten lassen sich kaum von den Substanzen trennen, deren Qualitäten sie sind. Farbe jedoch bewahrt sich die Eigenschaft des bloßen Erscheinens und bildet damit eine "Ordnung in unendlichen Nüancen« (GS VI, 110). Farbe erlaubt zahllose kontinuierliche Übergänge, die, so minimal sie auch sein mögen, umgehend offensichtlich sind. Deshalb kann Farbe als ein Medium erlebt werden, das fließende und kleinste Übergänge an sich vollzieht. Nicht die Mischung gesättigter Farben sondern die Betrachtung von Transparenzen gibt häufig die Möglichkeit, Unterschiede der Intensität, den Schimmer des Lichtes sowie die feinen und sich wandelnden Nuancen und beständigen Verschmelzungen zu erfahren. Seifenblasen, Schmuckstücke, Abziehbilder, die Laterna Magica ermöglichen den Wasserfarben gleich, wie Benjamin schreibt, eine "reine Anschauung in der Phantasie« (man beachte die Ausdrucksweise). Ihr "Zauber [hängt] [...] am farbigen Schein, am farbigen Glanz, am farbigen Strahl« (GS IV, 614). Aus diesen Erscheinungen hebt Benjamin den Regenbogen als "ein rein kindliches Bild« heraus - ein Bild, dessen Grenzen allein durch Farbe bestimmt sind: »In ihm ist die Farbe ganz Kontur» (GS VI, 110). ${ }^{4}$

4 Die verschiedenen Farben des Regenbogens und ihre geometrische Gestalt mögen uns dazu verleiten, die Farbordnung nach dem Modell der Harmonie zu verstehen (Newton hatte in der Tat versucht, eine Analogie herzustellen zwischen den sieben Farben, die er bei seinen Spektralexperimenten glaubte erkannt zu haben, und der Oktave in der Musik). Nun macht aber die Existenz des Regenbogens erst recht das ohnehin Offenbare deutlich: Während Farbe zur Erfahrungswelt gehört, setzen sich die musikalischen Harmonien nicht aus den Geräuschen, die wir hören, zusammen. Deshalb erregt Musik oft den Eindruck, nicht von dieser Welt zu sein. Ferner besagt die Möglichkeit, Farben auf einem Rad dazustellen, nicht,
Man könnte hier von der Erfahrung müheloser Grenzbeseitigung sprechen, von einer Dimension der Erfahrung, von der man fühlt, der Wandel in sei ihr in besonderem Maße möglich. Aber es ist ein Wandel ohne Zerstörung. "Schmerzlose Entstaltung " ${ }^{5}$ hat Benjamin dies genannt, in starkem Gegensatz zu Gefühlszuständen der Angst, Erhabenheit oder Ekstase, in denen wir uns als Welt in ihrer grenzenlosen Totalität Erfahrene vorstellen. In Bezug auf Farbe ist die Erfahrung der Verschmelzung eine solche fortwährende Auflösung, ohne aber in ihr der Versuchung zur Transzendenz zu erliegen. Die Stimmung der Farbe wird, so Benjamin, geschaffen, »ohne darum die Welt aufzugeben« (111).

Beziehungen, welche die Erfahrung farblich vervollständigen, tun dies nicht in Abhängigkeit von der Gesetzmäßigkeit der Erfahrung. Farbe beinhaltet ein Höchstmaß an Empfänglichkeit für Erfahrung, aber weder entspricht ihre Ordnung den Tatsachen der Erfahrung, noch ist sie deren bloße Reflektion. Farbe gehört der Erfahrung zu, ist zugleich aber von den kausalen, zeitlichen, räumlichen, begrifflichen und sogar metaphorischen Determinanten verschieden. Genau über diese ihre Selbstgenügsamkeit, Unterscheidungen innerhalb einer Ordnung zu ermöglichen, deren internes Bezogensein sowohl grenzenlos als auch unmittelbar ist, belehrt sie das Kind. ${ }^{6}$ "Aus der Erfahrung lernen« bedeutet uns hauptsächlich, widrigen Umständen begegnen zu müssen - sei es mit Hindernissen und Qualen beschäftigt zu sein; sei es mit der abstumpfenden Einübung von Gewohnheiten, die auf den Gesetzmäßigkeiten der Erfahrung beruhen. Meine Beschreibung der Entgrenzung auf dem Feld der Farben berücksichtigt möglicherweise weder die eine noch die andere und überführt Erfahrung in eine Micky Maus-Welt, in der es, wie Benjamin schreibt, sich nicht »lohnt", "Erfahrungen zu machen« (144). Da alles formbar ist, würde nichts lohnen, erinnert zu werden. Auch würde die Phantasie keine Gelegenheit finden, ihr Vermögen zur Assoziation ins Spiel zu bringen.

dass sie eine quasimathematische Ordnung bilden: »Farbe«, schreibt Benjamin, „verhält sich zu Optik nicht, wie Linie zu Geometrie« (GS VI, 109).

5 Vgl. Benjamins Fragment »Phantasie«: »Alle Entstaltung der Welt wird also in diesem Sinne eine Welt ohne Schmerz phantasieren, welche dennoch vom reichsten Geschehen durchflutet wäre." (GS III, 115).

6 Farbe ist von zentraler Bedeutung für eine Vielzahl von Bildungsidealen, die von den Ideen Rudolf Steiners bis zu denen des Bauhaus-Konzeptes reichen. 
Dennoch ist »Phantasie [...] nur durch [...] Anschauung von Farben [...] zu entwickeln« und »in Zucht zu halten« (110). Damit meint Benjamin nicht, Imagination sei ein schöpferisches Vermögen, das gegebenes Wahrnehmungsmaterial neu formiert. Stattdessen unterscheidet er eine solch formative Einbildungskraft von der Phantasie, die sich in der Beziehung des Kindes zur Farbe offenbart.

Phantasie ist kein formierendes Vermögen, sondern ein Medium der Deformation. Wenn Farbe als eine ihrer deutlichsten Manifestationen verstanden wird, lässt sich mit Phantasie etwas anderes verbinden als bizarre, groteske oder außerirdische Charaktere. Es wäre der mit Farbe verbundene Sinn einer "zusammenhängende[n] Anschauung der Phantasiewelt" (ebd.), der die eigene Deformierung verwirklichte. Die Phantasie würde die Welt nicht im Sinne der eigenständigen Identität raumzeitlich individuierter und voneinander unterschiedener Gegenstände erfassen, sondern - so befremdlich dies auch scheinen mag - als eine sich beständig deformierende, mithin nur sich gleichende Totalität. Die Deformation von Erfahrung wäre kein äußerlicher Wandel, dem Substanzen unterliegen. Sie würde stattdessen in der Vertiefung der inneren Zusammenhänge liegen, die der Erfahrung durch die Farbe ermöglicht wird. So gesehen wäre Farbe das Medium, das Phantasie und Wahrnehmung zusammenführt.

\section{Exkurs über das Erwachen aus dem Geschmack und dem Geruch der Kindheit}

An dieser Stelle nimmt meine Diskussion um die Farben und Geräusche der Berliner Kindheit einen kurzen thematischen Umweg, denn ich möchte die Sinnesräume von Geruch und Geschmack mit der Realisierung der Vergangenheit in der Erinnerung in Beziehung bringen. Wie in meiner Diskussion bereits deutlich geworden ist, besteht die ergreifende Macht der Bilder nicht darin, dass die gestaltlose Umgebung, für die sie stehen, vermöge eines narrativen Musters, durch Abfolge und Kausalität zusammengefügt worden wäre. Die Zusammenführung erfolgt vielmehr in der Erinnerung und führt zu einer imaginären, dem Traum verwandten Konfiguration. Die biographischen Umstände - zu einer bestimmten Zeit an einem bestimmten Ort zu sein - schaf- fen, um hier ein Wortspiel aus dem Passagen-Werk zu nutzen, nicht nur einen Zeitraum, sondern auch einen Zeit-traum (vgl. GS V, 491). Das bedeutet nicht, das Kind erfahre seine Kindheit in der Stadt wesentlich als Traum. Es bedeutet, dass in der Erinnerung die Umgebung anfänglich zutage tritt, indem sich - Träumen ähnlich - verschiedene Elemente zu Bildern zusammenfügen.

Weiter schreibt Benjamin, er halte es "für möglich, daß solchen Bildern ein eigenes Schicksal vorbehalten ist" (GS VII, 385), und deutet damit an, dass die Traumbilder eine weitere Zustandsänderung erfahren oder zur Wahrheit auskristallisieren werden. Angesichts der Traumkonfiguration der Erinnerung der Vergangenheit, mit der Benjamin befasst ist, nennt er deren nunmehriges Auskristallisieren in der Erfahrung "Erwachen«. Hier möchte ich kurz diese eigenartige Beziehung zur Vergangenheit, dem Gegenstück einer Zeit der Bedeutung, charakterisieren, die der Konstitution der Gegenstände der Erinnerung zugehört. Ich werde sie die Zeit der `Realisierung` der Bedeutung nennen, wobei ich auf die Doppeldeutigkeit von 'Realisierung anspiele: einerseits im Sinne von verwirklichen, andererseits als anerkennen bzw. klar erkennen.

Die Realisierung des Bedeutungsumfelds dessen, was erst einmal eine Traumkonfiguration darstellt, ist vielleicht etwas provokant gesagt eine Art Traumerfüllung. Dies mag erklären, warum Benjamin zu Anfang des mit "Wintermorgen" überschriebenen Abschnitts der Berliner Kindheit die Welt der Märchen heraufbeschwört: »Die Fee, bei der er einen Wunsch frei hat, gibt es für jeden." Der Wunsch, den Benjamin meint, kennt jedes Schulkind nur zu gut: Er besteht darin, "ausschlafen zu können«. Er habe diesen Wunsch "wohl tausendmal getan", schreibt Benjamin, "und später ging er in Erfüllung. Doch lange dauerte es, bis ich sie darin erkannte, daß noch jedesmal die Hoffnung, die ich auf Stellung und ein sicheres Brot gehegt hatte, umsonst gewesen war.« (397 f.).

Der bittersüße Geschmack, den die abschließende Bemerkung hinterlässt, in der man der Wahrheit ins Auge blickt, besagt m. E., dass die ironische Wunscherfüllung mehr als simple Ernüchterung sein kann, dass Erwachen nicht im Zunichtemachen von Hoffnungen bestehen muss. Um diesen intuitiven Gedanken zu rechtfertigen, müsste man sich mit der eigenartigen, hier beschriebenen Zeitstruktur befassen, erstens ganz zu Beginn der Wunschbildung, dann in der Beschreibung der Wunscherfüllung und schließlich in der 
zwischen Wunsch und Erfüllung etablierten Beziehung. Der Wunsch, schreibt Benjamin, »bildete sich in mir mit der Lampe, wenn sie am frühen Wintermorgen um halb sieben sich meinem Bette näherte und den Schatten des Kindermädchens an die Decke warf.« Das Kindermädchen öffnet die Ofentür und legt einen Apfel in die Ofenröhre. Das »rote[] Flackern auf der Diele«, in dem sich das "Gatter der Kamintür« abzeichnet, wie auch der "schaumige[] Duft« des Apfels, locken das Kind, ohne dass es ganz aufgewacht wäre, aus dem Bett. (Ebd.) Der Duft begleitet das Kind noch auf dem Weg zur Schule, aber erst als es sich auf der Schulbank niederlässt, bricht der Wunsch aus ihm heraus und wird geäußert. Die anfängliche Wunschbildung und dessen letztendliche Formulierung und Äußerung sind voneinander getrennt. Beide unterscheiden sich durch ungeformte Erfahrungen, die sowohl ein Substrat zur Anreicherung von Bedeutung liefern als auch eines, das der Gestaltung der Aura des Wunsches dient. Der Schatten, der Duft und der flackernde Widerschein des Lichts verschaffen dem Wunsch ein Nachleben, das der bloße Gedanke - "Ich wollte wirklich nicht zur Schule» -, wie intensiv und oft er auch erscheinen möge, nie zustande brächte.

Eine ähnliche Trennung der Momente besteht in Bezug auf die Erfüllung. Wenn Benjamin schreibt, der Wunsch sei »später [...] in Erfüllung« gegangen, obwohl es »lange dauerte [...], bis [er] [...] sie [die Erfüllung] darin erkannte, daß«... Dies lässt vermuten, dass die wirkliche Erfüllung des Wunsches nach Schlaf nun nicht mehr zur Debatte steht (weil er fraglos nicht mehr zur eigenen Glücksvorstellung gehört). Der Schwerpunkt der Anerkennung liegt jetzt auf der zeitlich und logisch eigenständigen Möglichkeit, eine bedeutungsvolle Verbindung zwischen Vergangenheit und Gegenwart herzustellen.

Die Hinterhältigkeit der allumfassenden Zeitlichkeit der Realisierung unterstreicht Benjamin, wenn er schreibt, dass "wenige [...] sich des Wunsches zu entsinnen [wissen], den sie taten; nur wenige [...] darum später im eignen Leben die Erfüllung wieder« erkennen (ebd.). Zwischen Wunsch und Erfüllung besteht eine grundsätzliche Diskontinuität; es fehlt an Zusammenhang in zeitlicher, kausaler und sogar in teleologischer Hinsicht. Darüber hinaus ist die Deformierung der Wunschbilder so zu verstehen, dass wir nicht mehr auf selbstverständliche Weise erkennen können, was eine Möglichkeit ihrer Realisierung wäre. Es ist somit eine List vonnöten, um in den gegenwärtigen Umständen das Scharnier zu erblicken, in welchem sich die Bedeutung der Ver- gangenheit bewegt, in dessen Betätigung die ungeformten Erinnerungen zu einer sinnvollen Einheit finden.

Man würde Gefahr laufen, Benjamins Leben zu romantisieren oder zu ästhetisieren, wenn man darin mehr als eine Allegorie sähe. Und dennoch liegt der entscheidende Aspekt der Allegorie nicht einfach darin, die Unterscheidung zwischen dem Erwachen der Realisierung und den Leistungen des Wissens zu treffen, sondern auch - vermöge dessen - die Möglichkeit einer bejahenden Einstellung angesichts von Versagen und den Enttäuschungen des Erwachsenseins. Der Geschmack des Apfels, so ließe sich sagen, muss nicht gleichgesetzt werden mit den (bekannten) Konsequenzen jenes Augenblicks im Garden Eden. Weder würde man die Hoffnungen und Wünsche der Vergangenheit als bloße Illusionen abtun wollen, noch sie mit ganzem Herzen akzeptieren und allein den Lebensweg bejahen, auf dem sie, so wie sie sind, verwirklicht werden können. So gestaltet Benjamin sein kleines Bild nach dem Muster einer aus Märchen bekannten Zwickmühle: Angesichts seiner Erfüllung bedauerten wir, den Wunsch jemals gehegt zu haben. Wir hatten sozusagen nicht realisiert, was das für eine Welt wäre, in der sich der Wunsch erfüllt hätte. Obwohl die Erfüllung des geäußerten Wunsches darin besteht, alle Zeit der Welt über schlafen zu können, so ist es doch, und das wird damit betont, der entstehende Bedeutungskontext - die Möglichkeit, das Leben nacherzählend gegen den Strich zu bürsten -, der die erwachende Realisierung dieser Vergangenheit konstituieren würde. Zu einem solchen Erwachen würde man nicht gelangen, wenn man einfach dem inneren, im Traumbild verkapselten Telos folgte. Stattdessen wäre es nötig, in seiner Entfaltung eine Möglichkeit zu finden, den Wunsch aufzuheben: auf dass man von seiner Erfüllung verschont bliebe.

Für diese Rettung der Vergangenheit ist mehr erforderlich als die übliche Tugend des Heldenmuts. Wie Benjamin schreibt, polarisiert das Märchen den traditionellen Mut des Helden in Untermut (d.h. List) und Übermut. Der Untermut ist als die List zu verstehen, die nötig ist, um die Schleichwege zu finden, die Gegenwart und Vergangenheit verbinden können. Übermut ist erforderlich, um die ernüchternde Interpretation zu bejahen, die die Vergangenheit in der Gegenwart erfährt. Das Modell wahrheitsgetreuer Erinnerung, das mit dieser Form der Bejahung der Vergangenheit korrespondiert, ist nicht das ihres Nacherlebens oder des emphatischen sich Identifizierens mit dem 
Selbst, das man einmal war. Benjamins Autobiographie ist so wenig ein regressiver oder sentimentaler Versuch, den Geschmack der Kindheit zurückzugewinnen, wie Prousts Leistung etwa darin bestanden hätte, sich den Geschmack der Madeleine zurückzuerobern. Wirkliche Einsicht lässt sich stattdessen nur dann erreichen, wenn sich die Vergangenheit im Raum gegenwärtiger Erfahrung verwandelt, wenn der Traum, in welchem das Material der Vergangenheit eine Bedeutung vermöge der Gegenwart aktualisierte, realisiert wird.

\section{Akustische Brechungen}

Welche Rolle spielen dann aber Geräusche in diesem Schema einer im Sinne von Traum und Erwachen gedeuteten Vergangenheit? Farben bilden, so könnte man behaupten, den Extremfall einer Wahrnehmung und Phantasie zusammenführenden Traumkonfiguration. Die Erfahrung der Welt mittels Farben bringt die Deformierung der erinnerten Erfahrung zum Vorschein. Farben sind die unverfälschte Manifestation der Verwandlung des Wahrnehmungsraums in einen Traumraum, das unverstellte Beispiel der Anschauung in der Phantasie. Geräusche hingegen verweisen in Erinnerung und Wirklichkeit auf das Erwachen. Vor dem Hintergrund der Diskussion um die Farbe wird das Eigentümliche der Geräusche, welche die Berliner Kindheit heraufbeschwört, erst recht deutlich. Eine Auflistung solcher für die Erinnerung bedeutsamen Laute bietet der mit »Die Mummerehlen« überschriebene Abschnitt:

Ich hauste wie ein Weichtier in der Muschel im neunzehnten Jahrhundert, das nun hohl wie eine leere Muschel vor mir liegt. Ich halte sie ans Ohr. Was höre ich? Ich höre nicht den Lärm von Feldgeschützen oder von Offenbachscher Ballmusik, nicht einmal Pferdetrappeln auf dem Pflaster oder die Fanfaren der Wachtparade. Nein, was ich höre, ist das kurze Rasseln des Anthrazits, das aus dem Blechbehälter in den Ofen fällt, es ist der dumpfe Knall, mit dem die Flamme des Gasstrumpfs sich entzündet, und das Klirren der Lampenglocke auf dem Messingreifen, wenn auf der Straße ein Gefährt vorbeikommt. Noch andere Geräusche, wie das Scheppern des Schlüsselkorbs, die beiden Klingeln an der Vorder- und Hintertreppe [...]. (GS VII, 417)

Es sind Geräusche wie etwa Vibrieren, Klingeln, Schlagen, Brechen, Klappern, Knallen, Klirren und Widerhallen. Warum sind es solche Geräusche, an die die Berliner Kindheit erinnert, wieso stellen diese die maßgeblich erinnerten dar?

Während Farben als Umgebung erfahren werden, in die das Kind versunken ist, die es aufnimmt, treten Geräusche zumeist vereinzelt auf und sind umgeben von Leere oder Stille. Sie sind von jeglicher Umgebung abgekoppelt, vom Umfeld getrennt. Die Grundfigur dafür, sich mit den Geräuschen der Erinnerung zu beschäftigen, ist das Hören der Echos aus einer leeren Muschel, weil die Lebensform, zu der sie gehören, sich zurückgezogen hat. Diese Bedingung der Leere oder umgreifenden Stille ist eine solche, die viele ihrer Merkmale bestimmt.

Beginnen möchte ich mit einem Charakteristikum, das vielen Geräuschen gemeinsam ist: dem Vibrieren. Hierbei nehme ich an, dass wir in diesem Geräusch selber eine Art Räumlichkeit verspüren. Die Vibration weist auf eine Art Geräuschhülle hin, als ob das Geräusch nicht eins mit sich wäre, sondern immer ein wenig mehr als es selbst, als ob es sich von sich abheben würde. Wenn wir dies aber nicht im Sinne der Ausbreitung des Schalls in der Luft verstehen, sondern dabei eher an die Übertragung von Geräuschen im Medium der Erinnerung denken, so lässt sich dieses Geräuschmerkmal als Ausdruck der Mitwirkung der Imagination verstehen, als auditives Gegenstück zu dem, was Benjamin den 'Schleier` nennt. Eine verschleierte Erscheinung ist eine solche, deren ganzheitlicher Sinn zweideutig ist. Es handelt sich um eine Wesenseinheit oder um eine Einheit mit sich selbst - nur verschleiert. Dieser immanente Doppelsinn ist charakteristisch für die Attraktivität von Schönheit, er charakterisiert sie als Schein. Schönheit ist, so Benjamin, verschleierte Wahrheit. Der Schleier der Imagination zeigt sich für Benjamin in Momenten der Aufregung, des Bebens und Zitterns - der Schleier hebt sich ab, wenn er wallt. Dessen akustisches Gegenstück wäre die Vibration. Das Vibrieren entspräche nicht dem Heben des Schleiers, sondern würde, stattdessen, dass man sich weiter in den lebendigen Schein versenkte, auf dessen Präsenz verweisen und somit eine erste Distanzierung bedeuten.

Sicherlich sind wirkliche Vibrationen in einigen Geräuschen zu finden, welche die Berliner Kindheit erwähnt (so die verschiedenen im Text verzeichneten Klingel- und Glockentöne). Was mir jedoch bedeutsamer als diese erinnerten Vibrationsgeräusche zu sein scheint, sind Geräusche, unter ihnen Worte, denen die Erinnerung die Qualität von Vibrationen verleiht. Einer 
dieser Fälle ist das Wort „Brauhausberg", das den Ort der Schmetterlingsjagd bezeichnet: „Die Luft, in der sich dieser Falter damals wiegte, ist heute ganz durchtränkt von einem Wort [...]. So zittert durch die schmetterlingserfüllte Luft das Wort `Brauhausberg ‘.» (393)

Bedingung einer solcherart vibrierenden Erscheinung ist, wie Benjamin schreibt, "[l] anges Verschwiegenwordensein", dass das "Wort [...] seit Jahrzehnten nie mehr mir zu Ohren oder über meine Lippen gekommen ist « (ebd.). Als ob der Klang nur dann nachhallen kann, wenn das Wort außer Gebrauch gesetzt worden ist und jedweden Bezug zu anderen Worten verloren hat. Ist das Wort nicht mit anderen Worten zu Sätzen verknüpft, lässt sich eine Beziehung zwischen ihm und dem Bild herstellen. Das Wort ist dabei allerdings nicht im Sinne eines Bilduntertitels zu verstehen oder als dem Bild nur hinzugefügt, sondern in der Weise, dass es das Bild mit den Vibrationen seines Klanges erfüllt. Die Resonanz, die der Klang des Wortes erzeugt, erfährt man, während sie das Umfeld erfasst - wie etwa in der Bildsymbolik der mit bunten Schmetterlingen erfüllten Luft. So entsteht eine nicht nach Maßgabe der Erfahrung arbeitende Entsprechung zwischen dem Register der Geräusche und dem der Bilder. Diese Entsprechung hat »Brauhausberg« verklärt und von all dem entlastet, was dieses Wort mit Bier assoziiert. (Hierbei sei angemerkt, dass Synästhesie für Benjamin keine Sache der empirischen Psychologie ist, sondern die Beziehungen zwischen den Erfahrungsdaten betrifft, während diese im Medium der Erinnerung verwandelt werden.) ${ }^{7}$

7 Für Benjamin erhält die Erfahrung von `Entsprechungen` in der Tat ihre Bedeutung aus ihrer Beziehung zur Struktur der Erinnerung. Sie entsteht, wie er in Bezug auf Baudelaire schreibt, im Medium der Erinnerung. Die Bildung solcher nichtsinnlichen Ähnlichkeiten um hier ein anderes Wort für die gleiche Sache zu benutzen - kennzeichnet die Realisierung der Vergangenheit im Medium der Erinnerung. "Es wäre ein Irrtum, die Erfahrung, die in den correspondances beschlossen liegt, als planes Gegenstück zu gewissen Experimenten zu denken, die man mit der Synaesthesie (dem Farbenhören oder Tonsehen) in psychologischen Laboratorien angestellt hat. Bei Baudelaire handelt es sich weniger um die bekannten Reaktionen, aus denen die schöngeistige und snobistische Kunstkritik so viel Wesens gemacht hat als um das Medium, in dem solche Reaktionen erfolgen. Dieses Medium ist die Erinnerung und sie war bei ihm von ungewöhnlicher Dichtigkeit. Die korrespondierenden Sinnesdaten korrespondieren in ihr; sie sind geschwängert mit Erinnerungen, die so dicht heranfluten, daß sie nicht aus diesem Leben sondern aus einer geräumigern vie antérieure herzustammen scheinen. Auf dieses Leben spielen die regards familiers an, mit der solche Erfahrungen den Betroffenen ansehen." (GS V, 464).
Dass Laute vibrieren können, steht im Zusammenhang mit der ihnen eigenen Diskretheit, Unterbrechung oder Vereinzelung. Sie sind schwerlich auf andere Laute bezogen. Aus diesem Grunde sind Melodien etwa der Tanzmusik à la Offenbach oder rhythmische Geräusche wie das Pferdegetrappel auf dem Kopfsteinpflaster für die Erinnerung weniger bezeichnend. Ferner dürfte anhand der Beziehung, die »Brauhausberg« mit der farbenprächtigen Landschaft eingeht, deutlich geworden sein, dass sich Laute den Bildern nicht einfach zuordnen oder diese begleiten. Zuweilen signalisieren sie sogar eine Unterbrechung im Fortgang des Gesehenen, deuten einen Wechsel an. So wird etwa im Abschnitt über das Kaiserpanorama ein Unterschied zwischen den Wirkungen der Begleitmusik im Kinofilm und dem Klingelton aufgemacht, der den Wechsel der Szenerie in der nunmehr veraltenden Apparatur ankündigt. ${ }^{8}$ Die musikalische Filmbegleitung stellt eine zu enge Beziehung zwischen den Registern von Klang und Bild, ein Zuviel an Entsprechungen zwischen beiden her - was ermüdend wirkt. Hingegen ist der Klingelton des Panoramas dem Aufwachen verbunden, einem Unterbrechen und Wachrütteln - unmittelbar, bevor der Apparat sich dann in Bewegung setzt.

Die alarmierende, störende Eigenart der Geräusche, ihr Vermögen, wachzurütteln, wie auch ihre Verwandtschaft mit einer Apparatur - all dies sind Themen des Abschnitts über das Telefon. Sein durchdringender Laut raubt nicht nur den Eltern die Mittagsruhe, sondern gleicht einem Alarmzeichen, das den Fortgang der Lebensweise einer ganzen Geschichtsepoche bedroht. Das Geräusch des Telefons signalisiert einen, die wahre Geburt auszeichnenden Zeitumbruch.

Es mag am Bau der Apparate oder der Erinnerung liegen - gewiß ist, daß im Nachhall die Geräusche der ersten Telefongespräche mir anders in den Ohren liegen als die heutigen. Es waren Nachtgeräusche. Keine Muse vermeldet sie. Die Nacht, aus der sie kamen, war die gleiche, die jeder wahren Geburt vorhergeht. Und eine neugeborne war die Stimme, die in den Apparaten schlummerte. (390)

8 Als ob der Vergleich zwischen dem Klingelton und der fortlaufenden Musik uns die beschleunigte Abfolge von Bildern im Kinofilm im Unterschied zum Bilderwechsel im traditionellen Panorama veranschaulicht. Man beachte, dass auch dies eine Beziehung von Geräusch und Bild darstellt, d. h. eine weitere Instanz von Synästhesie in der Erinnerung. 
Diese Zeilen verdichten ein Problemfeld von Klang und Erinnerung, das ich hier im Ganzen nicht darlegen kann. Immerhin möchte ich andeuten, wie es zu explizieren wäre: Die Telefongeräusche »Nachtgeräusche« zu nennen, legt nahe, dass Geräusche dieser Art nicht einem gegebenen Kontext entstammen, dass sie nicht Teil der Lebenswelt oder Existenzweise sind, in der sie vernommen werden. Stattdessen gleichen sie den Sternen, die nachts vor dem Hintergrund der dunklen Himmelsleere sichtbar werden. Es handelt sich um Geräusche, deren Bedeutsamkeit der Zukunft gehört. »Keine Muse vermeldet sie« heißt: Sie sind nicht neu in der Weise, in der ein künstlerisches Gebilde originär ist. ${ }^{9}$ Die Tatsache, dass kein Genie an ihrem Erscheinen beteiligt ist, erklärt sich gewiss unter anderem aus der Tatsache, dass Geräusche dieser Art technisch erzeugt und übertragen werden. Wie es keine Muse der Fotografie gäbe, gibt es auch keine fürs Telefon. Ein weiterer bemerkenswerter Fall, in dem Benjamin das Auftreten von Musen leugnet, ist das Übersetzen. Wenn uns das letztere als Modellfall des Nichtinspirierten gilt, ließe sich sagen, die Bedeutung der Geräusche, ihre Neuheit gehöre ihrem zukünftigen Leben oder offenbare sich in ihm.

Dieser Gedanke scheint mir für die Entweder-oder-Struktur im Eröffnungssatz des fraglichen Abschnitts wesentlich zu sein. Allerdings könnte man dazu neigen, diesen folgendermaßen zu interpretieren: Entweder haben sich die Geräte weiterentwickelt und Telefongespräche klingen aus diesem Grunde anders als in der Vergangenheit oder die Telefongespräche der Vergangenheit werden im Medium der Erinnerung entstellt und klingen aus diesem Grunde anders als die in der Gegenwart erfahrenen. Benjamins Formulierung nötigt uns, wie ich glaube, dazu, die Dichotomien von subjektiv und objektiv, Gegenwart und Vergangenheit zu vermeiden. Stattdessen müssen wir erkennen,

9 Man bemerke, dass Farben in der Welt des Kindes in ihrer Buntheit erfasst werden, nicht aber als inspiriert oder künstlerisch. Es ließe sich einwenden, dass Ausmalen eine mechanische Angelegenheit ist und aus diesem Grunde für die Kindererziehung von geringerem Wert als jene Aktivitäten, welche die Kreativität befördern. Möglicherweise verbirgt aber gerade diese Betonung der Kreativität die charakteristische Funktion der Imagination in der Welt der Kinder. In der Tat unterscheidet eben die Erkenntnis, dass man in Bezug auf Farben nicht aktiv sein kann, diese Arten rezeptiver Phantasie vom schöpferischen Vermögen der Erwachsenen und verleiht ihnen ihren Wert. Für Benjamin ist »das Austuschen eine reinere pädagogische Funktion als das Malen« (GS VI, 111). dass sich die Identität der Vergangenheit insofern ändert, als die Gegenwart deren Potenziale offenbart. Im Gegenzug erschließt sich die Gegenwart im Zuge der Anerkennung ihrer Verwandtschaft mit der Vergangenheit. Die wahre Geburt ist anders gesagt nicht an einer Quelle ausfindig zu machen. Sie kann als solche nur aus der Distanz (an)erkannt werden (was schon von Benjamins berühmter Definition des Ursprungs her deutlich sein dürfte). In der Realisierung des Erweckungspotentials der Technologie vermag deren Vergangenheit als wahre Geburt erscheinen, das bedeutet als eine neue Gestalt der Natur.

Nachtgeräusche oder Nachtklänge treten genau dann in aller Deutlichkeit hervor, wenn die Farbe verschwindet. Im mit »Der Mond « überschriebenen Abschnitt arbeiten Entfärbung und Echo gemeinsam an der Erschaffung eines unheimlichen Gefühls von Weltverlorenheit. Entfärbung meint nicht einfach Dunkelheit sondern erheischt, Dinge in einem anderen Licht zu sehen. Im blassen Schein, den der Mond nachts ins Zimmer und auf die Porzellankannen, marmornen Oberflächen und cremefarbenen Becken des Waschgeschirrs wirft, entfärbt sich für das Kind die Welt. Während die Stimmung der Farbe eine des sich Versenkens ist, schafft die Entfärbung eine Trennung von der Welt. "Wenn [der Mond] im Zimmer stand und ich erwachte, so war ich ausquartiert, denn es schien niemanden als ihn bei sich beherbergen zu wollen.« Als ob das Kind eine "Nebenerde« erwägt, in welcher der Platz, den es als ihm zugehörig erkennt, schon durch das Kind besetzt ist, das es einst war. »Ich mußte mich darein begeben«, schreibt Benjamin. »Trat ich dann ans Bett, so war es immer mit der Angst, mich selbst schon darin ausgestreckt zu finden." Die das Kind erschöpfende Sorge verschafft ihm die erste Begegnung mit der Metaphysik: Nichts war "mehr vorhanden [von der Welt] als eine einzige verstockte Frage. Sie lautete: warum denn etwas auf der Welt, warum die Welt sei?« (472 f.) In der Entfärbung verliert sich das Gleichgewicht zur Welt, als ob man das Gewebe verlöre, durch das man ihr zugehört. Diese Trennung von der Lebenswelt und die skeptischen Zweifel, die mit dieser Entzweiung verbunden sind, finden ihr Gegenstück in den Geräuschen dieses Textabschnitts.

Dass Laute vibrieren können, wurde schon hervorgehoben. Hier nun wird diese Eigenschaft auf den Übertragungs- oder Kommunikationsmodus der Geräusche bezogen, auf deren mögliche Vervielfältigung und Existenz unabhängig von der Lautquelle. In der Tat pflanzen sich Laute nicht einfach fort. 
Stattdessen schafft die Vibration Kommunikation durch den Kontakt direkt aneinander liegender Gegenstände, so dass sich der Klang über verschiedene Medien vom einem zum anderen überträgt. "Wenn ich aus meinem Bett stieg, klirrten [die Porzellankannen], und dieses Klirren pflanzte sich auf dem marmornen Belag des Waschtischs zu seinen Schalen und Näpfen fort." (427) Die Kommunikation der Geräusche ruft gewissenmaßen ein materielles Echo hervor und mit ihm die Vorstellung, alle Geräusche seien Wiederholung. "Das Glucksen dieses Wassers, das Geräusch, mit dem ich erst die Karaffe, dann das Glas abstellte - alles schlug an mein Ohr als Wiederholung." (Ebd.) Das Echo hat teil an der unheimlichen Verdopplung der Welt selber, die Gegenstand dieses Abschnitts ist.

Das Echo verschafft ein Gefühl der Ablösung von der Welt und verweist auf die Möglichkeit eines unabhängigen Fortlebens. Für Benjamin ist es ein Bild der Übertragung und des Fortlebens der Vergangenheit. Zwar ist es ein Bild der Verdopplung, im Unterschied aber zur Reflexion ist hiermit keine Verdopplung allein im Raum gemeint, sondern auch eine in der Zeit. Ferner beinhaltet das Echo den Aspekt der Gebrochenheit, denn Kommunikation ist gebrochen, weil sie über verschiedene Medien unterschiedlicher Dichte erfolgt. Auf den Unterschied zwischen Reflexion und Gebrochenheit komme ich hier deshalb zu sprechen, weil er m. E. von zentraler theoretischer Bedeutung für Benjamin ist. Reflexion ist ja der Schlüsselbegriff für das Verständnis der Kunstkritik in der deutschen Romantik, die eine der Formen darstellt, die literarischen Werken ein Fortleben verschafft. ${ }^{10}$ Andererseits ist Gebrochenheit unentbehrlich für das Nachdenken über das Erbe Goethes und die Darstellungsweise von Urbildern. Darüber hinaus ist er ein Schlüsselbegriff für Benjamins Theorie der Übersetzung.

Das letzte Merkmal der Geräusche, das ich hier ansprechen möchte, betrifft die Möglichkeit, die es der Konzentration eröffnet. Ihrer Verbreitung und Echowirkung zum Trotz lassen sich Geräusche auch als gesammelt, begrenzt und umschlossen erfahren. In diesem Sinne schreibt Benjamin, dass die sonn-

10 Reflexion entstammt als Begriff der reflektierenden Urteilskraft der Kritik der Urteilskraft von Kant und wird von der Romantik über die Vermittlung Fichtes angeeignet. Für Benjamins Dissertation über den Begriff der Kunstkritik in der deutschen Romantik ist er von grundlegender Bedeutung. tägliche akustische »Glockenfracht« der das Haus umgebenden Kirchen in der Loggia bis zum Abend »aufgestapelt« blieb. (413) Noch konzentrierter werden Klang und Ausblick im Abschnitt über den Besuch in Tante Lehmanns Haus erfasst. Deren brüchige, spröde, gläsern klingende Stimme war das Pendant zum Miniatur-Bauwerk. Das steckte in einem gläsernen Kasten und wurde dem Kind bei jedem Besuch zur Unterhaltung vorgeführt. Die Tante »trug Sorge, daß man den großen Glaswürfel vor mich stellte, der ein ganzes lebendiges Bergwerk in sich schloß, worin sich kleine Knappen, Hauer, Steiger mit Karren, Hämmern und Laternen pünktlich im Takte eines Uhrwerks regten" (399). Miniatur ist Konzentration, das Gegenteil von Ausbreitung. Sie ist, so ließe sich sagen, die verkürzte, monadische Zusammenfügung und Darstellung von Weite.

Man könnte darüber spekulieren, ob Benjamins Lärmpsychose, die sein Konzentrationsvermögen beeinträchtigte, mit verantwortlich dafür war, dass er nach Wegen suchte, sein Gehör zu konzentrieren. Jedenfalls sind die Geräusche, welche in der Berliner Kindheit als die unangenehmsten geschildert werden, genau jene, die eine diffuse Kulisse bilden. "Im Schwimmbad», so schreibt er, "widerte mich der Stimmenlärm, der sich in das Brausen der Leitung mischte, am meisten an. Er drang schon aus der Vorhalle«. ${ }^{11}$ Wichtig ist ferner, dass er die Auswirkungen dieser Geräusche in eine Analogie zur Göttin Lethe stellt, die »darauf aus war, uns an die Brust zu legen und aus den kalten Kammern uns zu tränken, bis dort oben [d.i. außerhalb des Bades] nichts mehr an uns erinnern werde« ( $415 \mathrm{f}$.).

Des Weiteren möchte ich auf die Bedeutung verweisen, die Benjamin Goethes Neuer Melusine zugemessen hat - einem Märchen, das von einem Jüngling erzählt, der sich in eine winzig kleine Prinzessin verliebt. Alles an ihr ist liebenswert, zart und vollendet. Dennoch besteht der unstete, zu Ausschweifungen neigende Jüngling keine der Prüfungen, die sie ihm stellt, damit er ihr

11 Gershom Scholem zitiert aus einem Brief, in dem Benjamin über seine Lärmpsychose berichtet: "Ich habe viel gearbeitet, aber dabei, sei es von wirklichem Lärm, sei es von übergroßer Konzentrationsanstrengung wieder einmal meine Lärmpsychose bekommen, so daß ich völlig herunter gekommen bin, jede Stimme [...] mich zur Raserei bringt und ich erwägen muß, nur nachts zu arbeiten, was auch viel Mißliches mit sich bringt. Haben eigentlich andere Leute Ruhe oder nicht?" Gershom Scholem: Walter Benjamin - die Geschichte einer Freundschaft, Frankfurt a. M. [Suhrkamp] 1975, S. 143). 
seine Liebe bekundet. Selbst als er mit ihr geradewegs ins Königreich der Zwerge geht, ist er unfähig, sich den Erfordernissen seiner Miniaturexistenz angemessen zu zeigen und ihnen nicht auszuweichen. Das für den Jüngling am meisten Abstoßende ist bezeichnenderweise die Musik. Vermutlich ist es die akustische Konzentration, welche die Musik bietet, die im größtmöglichen Gegensatz zum ausschweifenden, um nicht zu sagen sentimentalen Charakter des Jünglings steht.

Abgesehen von den Märchen gibt es eine systematische Rechtfertigung dafür, die Musik im Sinne von Sammlung und Konzentration zu begreifen. Schon früh hatte Benjamin den Zyklus der im Gefühlsleben des Wortes auftretenden Verwandlungen verzeichnet und deren wesentliche Abschnitte als den Laut der Natur, dessen Hemmung und Widerhall in der Trauer und schließlich, vermöge ihrer Beziehungen, die Sammlung des Widerhalls im reinen Laut des Gefühls bestimmt. ${ }^{12}$ Musik ist das unverfälschte Prinzip des

12 Benjamins frühe Abhandlung "Über Sprache überhaupt und die Sprache des Menschen" (1917) bildet den Kontext, um sowohl die Frage nach dem Wesensverhältnis des Naturlauts zur Musik als auch jene nach dem mimetischen Charakter der Sprache in einem umfassenderen linguistischen oder philosophischen Begründungsammenhang zu stellen. Die Abhandlung beginnt mit der Behauptung, es gebe »kein Geschehen oder Ding weder in der belebten noch in der unbelebten Natur, das nicht in gewisser Weise an der Sprache teilhätte, denn es ist jedem wesentlich, seinen geistigen Inhalt mitzuteilen" (GS II, 140 f.). Das "geistige Wesen« suche sich verständlich zu machen, es strebe oder trachte aus sich heraus nach Ausdruck. Wenn die Verwirklichung dieses Potenzials im vollendeten Ausdruck des geistigen Wesens besteht, dann ist das Telos der Wesenheiten Bedeutung oder Verständlichkeit. Ohne Mitwirkung des Menschen ist dieses Ziel nicht zu erreichen. Die Zusammenführung der Sprache der geistigen Wesenheiten und der Sprache des Menschen bringt den Menschen in die einzigartige Position, durch seine Sprache das Ausdruckspotential der natürlichen Dinge umzusetzen oder zu verwirklichen. Man könnte damit auch sagen, der Mensch habe kein eigenes Wesen. Stattdessen bestände seine Aufgabe darin, den Wesenheiten oder der Welt Ausdruck zu verleihen. Mit der Fähigkeit des Menschen, die Natur zu benennen, d. h. ihre Bedeutung in der Sprache zu artikulieren, ist, so Benjamin, dem in der Schöpfung liegenden Streben nach Ausdruck die Möglichkeit auf Verwirklichung gegeben: »Indem er die stumme namenlose Sprache der Dinge empfängt und sie in den Namen in Lauten überträgt, löst der Mensch diese Aufgabe." (GS II, 151) Benjamin sieht im Sündenfall den fundamentalen Riss, der die Beziehung des Menschen zur Sprache zunichte macht. Der Mensch scheitert an der adamitischen Aufgabe, der Schöpfung Namen zu geben, und wendet sich hauptsächlich der Kommunikation propositionaler Gehalte zu, d. h. den Urteilen. Es ist die propositionale Artikulation von Bedeutung - statt die Ausdrucksrealisierung der natürlichen Wesenheiten zu sein -, welche dieses Bestreben der Kreatur hemmt. Handelt es sich beim
Gefühls, so wie es sich auf dieser letzten Stufe von Sammlung und Konzentration darstellt. Es ist nicht meine Absicht, diesen Gedanken hier im Einzelnen darzulegen. Stattdessen möchte ich nur auf den Unterschied verweisen, der zwischen einer Auffassung besteht, die das Musikalische entweder aus der Idee der Natur begreift oder im letztlich Ekstatischen findet - im Wesentlichen wäre dies eine Identifikation mit dem dionysischen Element der Musik à la Nietzsche -, und einer Auffassung, die wie Benjamins das Musikalische als nüchterne Konzentration bestimmt. Überraschen kann diese Gegenüberstellung eigentlich nicht, wenn man an Benjamins systematischen Widerspruch zu Nietzsche denkt, der insbesondere deutlich wird, wenn er das Trauerspiel gegen die Tragödie stellt. Von Interesse dürfte hingegen sein, dass dieser Gegensatz seinen Widerhall in der Berliner Kindheit findet. Zumindest möchte ich in dieser Weise den Abschnitt "Zwei Blechkapellen« interpretieren, den einzigen der Schrift, der sich der Musik widmet.

Prägnant wird die im Titel angedeutete Dualität durch die unterschiedliche Verortung zweier Musiken: der Marschmusik des Militärorchesters in der Lästerallee des Zoo und dem Wiener Walzer, der die Schlittschuhläufer rund um die Rousseau-Insel begleitet. Zugleich ist das auch die jahreszeitliche Entgegensetzung einer Musik des Sommers und einer des Winters; einer Musik, die

Benennen um die linguistische Form, in der kreatürliche Stimme und Bedeutung zusammenfinden, so verkehren sich im Versagen an der Aufgabe, Dinge zu benennen - d. h. beim Sündenfall der Sprache ins Urteil -, Stimme und Bedeutung zu dialektischen Gegensätzen. Obwohl sie keine Verwirklichung von Bedeutung darstellt, lässt sich Musik so verstehen, dass sie diese Entgegensetzung im Gefühl überwindet. In diesem Sinnen besitzt sie für Benjamin eine Endgültigkeit, in der sich ihre rettende Kraft bekundet. Dies zeigt sich in seiner Diskussion des Verhältnisses von Sprache und Bedeutung in Bezug auf das Trauerspiel: „Es gibt ein reines Gefühlsleben des Wortes, in dem es sich vom Laute der Natur zum reinen Laute des Gefühls läutert. Diesem Wort ist die Sprache nur ein Durchgangsstadium im Zyklus seiner Verwandlung und in diesem Wort spricht das Trauerspiel. Es beschreibt den Weg vom Naturlaut über die Klage zur Musik. [...] [Das Trauerspiel] ist Natur, die nur um die Reinheit ihrer Gefühle willen ins Fegefeuer der Sprache steigt [...]. Denn es ist das Trauerspiel nicht der sphärische Durchgang des Gefühls durch die reine Welt der Worte mündend in Musik zurück zur befreiten Trauer des seligen Gefühls, sondern mitten auf diesem Wege sieht sich die Natur von Sprache verraten und jene ungeheure Hemmung des Gefühls wird Trauer." (GS II, 138) Vgl. dazu auch meine Abhandlung: "On the Musical Gathering of Echoes of the Voice. Walter Benjamin on Opera and the Trauerspiel«, in: Opera Quarterly 21 (2005), S. 631-646. 
sich »schwül und lockend im Laub- und Zeltdach wiegte», und einer anderen, »die blank und schmetternd in der kalten Luft wie unter einem dünnen Glassturz stand « (428). ${ }^{13}$ (Somit liegt darin auch eine Parallele zur Dualität der Farben des Sommers und des Winters wie auch zur Dualität des Schmetterlings und des Otters.) In beiden Fällen verwandelt die Musik die Körperempfindung, die Körperbewegungen wie auch das Wahrnehmungsvermögen des Körpers.

Im ersten Teil klingt das Militärorchester, das in der Nähe der Tierkäfige spielt, so entmenschlicht wie das Lautgetöse des Schwimmbads. Die Musik mischt sich mit den Rufen und Gerüchen der Tiere. Pauken und Schlagzeug werden als rhythmisches Getrommel erfahren, das sich der Kulisse der Tierschreie anverwandelt, während die Militärmusik in ihrer Brutalität sogar die Art und Weise beeinflusst, wie sich Sehen und Auratisches verflechten. Dieser Mangel an Grenzziehung zwischen menschlicher `Musikı und tierischen `Geräusch wie auch jener zwischen den verschiedenen Sinnesräumen findet seinen weiteren Widerhall in der Schwierigkeit des Jungen zur Selbstkontrolle, seine erotischen Regungen zu verbergen: „Und derart angestrengt war sein Bestreben, weder im Tonfall noch im Blick sich zu verraten, daß er von der Vorübergehenden nichts sah.« (Ebd.) Von der Innigkeit des erwiderten Blicks weiß, so ließe sich sagen, diese Art Musik gar nichts.

Diese ausschweifende Musik steht im Gegensatz zur kühlen Wahrnehmung klarer Grenzen, die sich verbindet mit der zweiten Blechkapelle, welche auf der Rousseau-Insel aufspielt. Jene Wahrnehmung von klanglicher Konzentration und Zurückhaltung, wenn nicht gar Miniaturisierung, findet im Gedankenbild des Glassturzes ihren Ausdruck. Wie der Glaskasten das MiniaturBergwerk enthält, birgt er die Musik. Zugleich verbindet sich mit dieser ein gewandeltes Sehen, die Möglichkeit einer Überschau, eines allumfassenden Blicks. Während auf der Lästeralle der Blick vor Verzückung sein Objekt verliert, hat man um die Rousseau-Insel herum die Möglichkeit distanzierter Überschau, einer Aufmerksamkeit, dem Blick von den Sitzbänken, die um das

13 Benjamin verfasste die Berliner Kindheit während seiner Aufenthalte auf Ibiza. Er schrieb dort u. a. auch einen mit "In der Sonne« betitelten Text, der um die Möglichkeit konzentrierten Denkens unter südlichen Temperaturen kreist. Geräusche, insbesondere Summen und Rauschen, sind für diesen Text von zentraler Bedeutung.
»Rondell der Buddelplätze« angeordnet sind (ebd.). Die Insel selbst dient wie ich annehme - hier als ein Symbol für die Möglichkeit einer konzentrierten Existenz. Diese spezifische Insel im Berliner Tiergarten ist nach der »Ile des peupliers« bei Ermenonville benannt, auf welcher Rousseau zuerst begraben wurde. Ermenonville ist auch der Ort, an dem Rousseau die letzten Monate seines Lebens verbrachte. Hier schrieb er seine Träumereien eines einsamen Spaziergängers, deren berühmter fünfter Spaziergang sich dem der Besinnung zuträglichen Dasein auf der Petersinsel im Bieler See widmet.

Die bedeutsamste Verwandlung aber, welche durch die Musik herbeigeführt wird, betrifft die Körperwahrnehmung. Die mehr als menschliche Anmut und Schnelligkeit, zu welcher das Eislaufen der menschlichen Bewegung verhilft, übersetzt sich in den normalen, aber nunmehr von der Last der Schlittschuhe befreiten Gang. Auf dem Weg nach Hause folgt die Musik dem Jungen, dem es ist, »als wüchsen Flügel« ihm »an beiden Sohlen« (ebd.). Während die Sommermusik den Menschen durch ihre Ähnlichkeit mit dem Tier entstellt, lässt sich der Verweis auf die an den Sohlen wachsenden Flügel als Hebung des menschlichen Vermögens verstehen, als Bild des Boten Hermes, eine höhere Seinsweise verkündend. Als die Musik den Jungen auf dem Nachhauseweg begleitet, zeigt sich, dass sie nicht nur dem Eislaufen dienlich ist. Aber sie hat hier auch nicht das Tempo bloßen Gehens, sondern begleitet ein solches, das sich vom alltäglichsten, banalsten oder eintönigsten zum festlichen verwandelt hat.

Übersetzung aus dem Englischen von Veit Friemert 